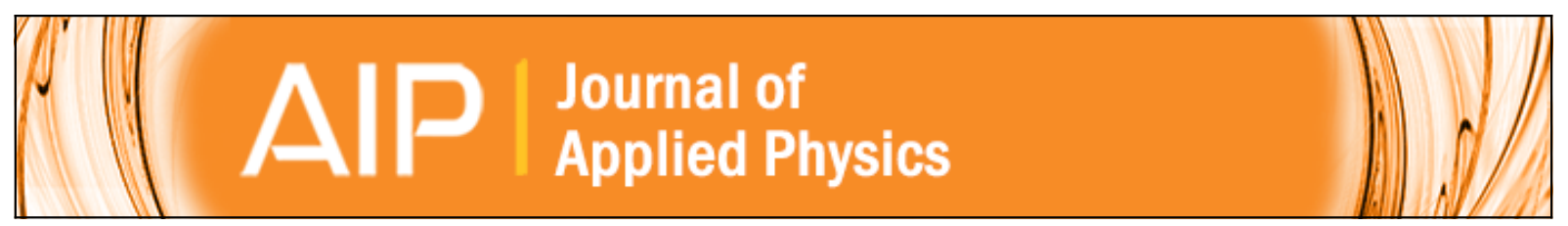

\title{
Switching ultrastrong light-matter coupling on a subcycle scale
}

R. Huber, A. A. Anappara, G. Günter, A. Sell, S. De Liberato, C. Ciuti, G. Biasiol, L. Sorba, A. Tredicucci, and A. Leitenstorfer

Citation: Journal of Applied Physics 109, 102418 (2011); doi: 10.1063/1.3578341

View online: http://dx.doi.org/10.1063/1.3578341

View Table of Contents: http://scitation.aip.org/content/aip/journal/jap/109/10?ver=pdfcov

Published by the AIP Publishing

\section{Articles you may be interested in}

Ultrastrong light-matter coupling in electrically doped microcavity organic light emitting diodes

Appl. Phys. Lett. 104, 233303 (2014); 10.1063/1.4882422

Thermo-optic plasmo-photonic mode interference switches based on dielectric loaded waveguides

Appl. Phys. Lett. 99, 241110 (2011); 10.1063/1.3670500

Liquid crystal-based dielectric loaded surface plasmon polariton optical switches

J. Appl. Phys. 110, 093102 (2011); 10.1063/1.3658247

Nonlinear switching in arrays of semiconductor on metal photonic wires

Appl. Phys. Lett. 98, 111104 (2011); 10.1063/1.3565167

Influence of the excitonic broadening on the strong light-matter coupling in bulk zinc oxide microcavities J. Appl. Phys. 108, 043508 (2010); 10.1063/1.3476553

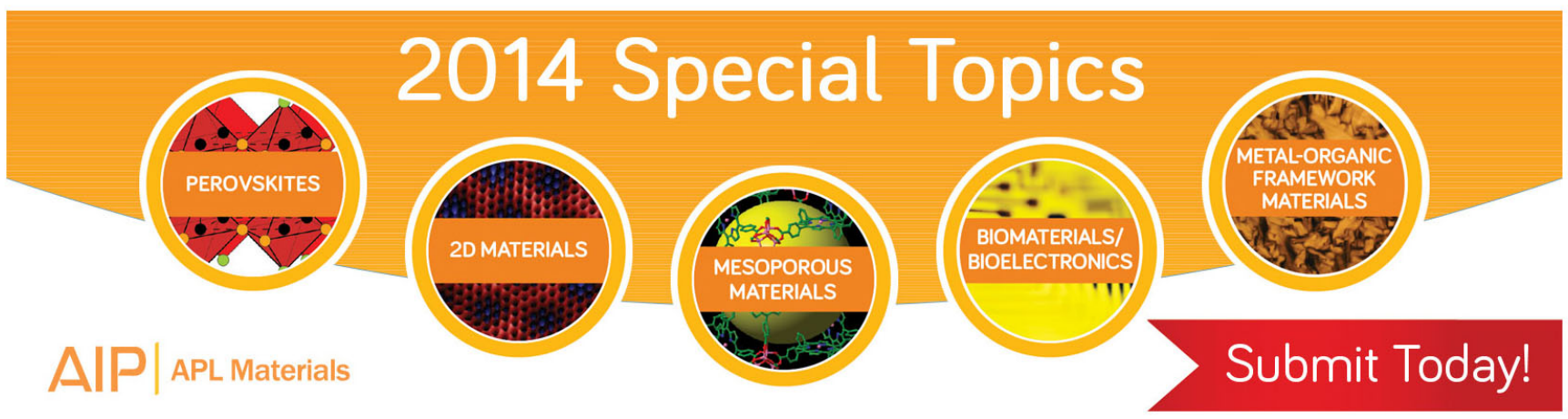




\title{
Switching ultrastrong light-matter coupling on a subcycle scale
}

\author{
R. Huber, ${ }^{1, a)}$ A. A. Anappara, ${ }^{1,2}$ G. Günter, ${ }^{1}$ A. Sell, ${ }^{1}$ S. De Liberato,,${ }^{3}$ C. Ciuti, ${ }^{3}$ G. Biasiol, ${ }^{4}$ \\ L. Sorba, ${ }^{2,4}$ A. Tredicucci, ${ }^{2}$ and A. Leitenstorfer ${ }^{1}$ \\ ${ }^{1}$ Department of Physics and Center for Applied Photonics, University of Konstanz, 78457 Konstanz, Germany \\ ${ }^{2}$ Laboratorio NEST, CNR-INFM and Scuola Normale Superiore, I-56126 Pisa, Italy \\ ${ }^{3}$ CNRS and Universite Paris Diderot-Paris 7 and Ecole Normale Superieure, 75205 Paris, France \\ ${ }^{4}$ Laboratorio Nazionale TASC CNR-INFM, 34012 Trieste, Italy
}

(Received 17 July 2010; accepted 28 December 2010; published online 31 May 2011)

\begin{abstract}
Intersubband cavity polaritons in a quantum well waveguide structure are optically generated within less than one cycle of light by a 12-femtosecond near-infrared pulse. Mid-infrared probe transients trace the nonadiabatic switch-on of ultrastrong light-matter coupling and the conversion of bare photons into cavity polaritons directly in the time domain. Future perspectives of room-temperature subcycle control of ultrastrong electron-photon interaction are discussed. (ㅇ) 2011 American Institute of Physics. [doi:10.1063/1.3578341]
\end{abstract}

Photonic microcavities have been exploited to enhance and tailor the interaction of light with specific elementary excitations. Optical resonators coupling to intra-atomic transitions, ${ }^{1,2}$ excitonic interband resonances, ${ }^{3,4}$ or intersubband excitations in semiconductor quantum wells have been intensely investigated. ${ }^{5-7}$ In the strong coupling regime, a photon may be absorbed and spontaneously reemitted by an elementary excitation many times before dissipation becomes effective. This process gives rise to new eigenstates of mixed light-matter character, so-called cavity polaritons. The characteristic energy anticrossing, known as vacuumfield Rabi splitting $2 \Omega_{R}$, is a direct measure of the strength of light-matter interaction.

Recently intersubband resonances of semiconductor quantum wells (QW) hybridized with the mid-infrared photon mode of a planar waveguide have entered a new regime of ultrastrong interaction, where $\Omega_{R}$ amounts to a significant fraction of the bare eigenfrequencies $\omega_{12}$ themselves. ${ }^{5,8-11}$ Microwave resonances in superconducting circuits have followed up reaching ultrastrong coupling. ${ }^{12,13}$ In this extreme limit, a theoretical description of the coupling of the intersubband resonance with the vacuum field of the resonator has to go beyond the rotating wave approximation (RWA). The resulting squeezed quantum vacuum is expected to give rise to a variety of novel quantum electrodynamical (QED) effects. ${ }^{14,15}$ In particular, ultrafast switching of the Rabi frequency $\Omega_{R}$ has been predicted to release correlated photon pairs out of the quantum vacuum, reminiscent of the intriguing, yet unobserved dynamic Casimir effect. ${ }^{14}$ Nevertheless, nonadiabatic phenomena - classical or QED in nature remained an academic curiosity since there has been no laboratory capable of controlling light-matter interaction on a subcycle time scale.

Here, we report an all-optical pump-terahertz probe scheme for the first implementation of femtosecond control of ultrastrongly coupled cavity polaritons. ${ }^{16}$ The experiment exploits latest techniques of broadband terahertz photonics capable of generating well-defined single cycles of light,

${ }^{\text {a)} E l e c t r o n i c ~ m a i l: ~ r u p e r t . h u b e r @ p h y s i k . u n i-r e g e n s b u r g . d e . ~}$ whose oscillating electric field is directly monitored with a subcycle resolution. ${ }^{16-23}$ This approach allows us to map out how a coherent population of bare photons is abruptly converted into cavity polaritons. Exciting new quantum phenomena that may become accessible by this approach will be discussed.

The idea of our experiment is based on intersubband transitions in semiconductor QWs, as sketched in Fig. 1. Our sample contains 50 identical, undoped GaAs QWs separated by $\mathrm{Al}_{0.33} \mathrm{Ga}_{0.67} \mathrm{As}$ barriers. The electronic wave functions are quantized along the growth direction forming subbands. These are unpopulated in thermal equilibrium. Radiative transitions between subbands of quantum number $n=1$ and $n=2$ are activated if optical excitation promotes electrons from the valence into the conduction band. The intersubband resonance features a narrow absorption line centered about a photon energy of $\hbar \omega_{12}=113 \mathrm{meV}$ (corresponding to a wavelength of $\lambda=11 \mu \mathrm{m}$ ) and a strong dipole moment oriented along the growth direction. ${ }^{24}$ The multi-QW structure is designed as a planar step index waveguide for midinfrared light. ${ }^{5}$ Radiation is confined between a top-cladding $\mathrm{Al}_{0.33} \mathrm{Ga}_{0.67} \mathrm{As}$-air interface $\left(n_{\text {air }}=1, n_{\mathrm{AlGaAs}}=3.1\right)$ on one side and a low refractive index AlAs layer $\left(n_{\mathrm{AlAs}}=2.9\right)$ on the other. The effective thickness of the entire waveguide is chosen to be $\lambda / 2$ at an internal angle of propagation of $\theta=65^{\circ}$. Photon modes with electric field components in growth direction (TM polarization) may resonantly couple to intersubband transitions provided the subbands are populated.

The Rabi frequency is known to scale with the electron sheet density $N_{e}$ in level $|1\rangle$ as ${ }^{14}$

$$
\Omega_{R}=\left(\frac{2 \pi e^{2}}{\varepsilon_{0} \varepsilon_{\infty} m_{0} L_{\mathrm{cav}}^{\mathrm{eff}}} N_{e} n_{\mathrm{QW}}^{\mathrm{eff}} f_{12} \sin ^{2} \theta\right)^{1 / 2},
$$

where $\varepsilon_{0}$ and $\varepsilon_{\infty}$ are the vacuum and the background dielectric constant, respectively, $L_{\mathrm{cav}}^{\text {eff }}$ the effective thickness of the cavity photon mode, $n_{\mathrm{QW}}^{\text {eff }}$ the effective number of quantum wells, $f_{12}$ the oscillator strength of the intersubband transition, and $m_{0}$ the free electron mass. In previous work, $N_{e}$ was 


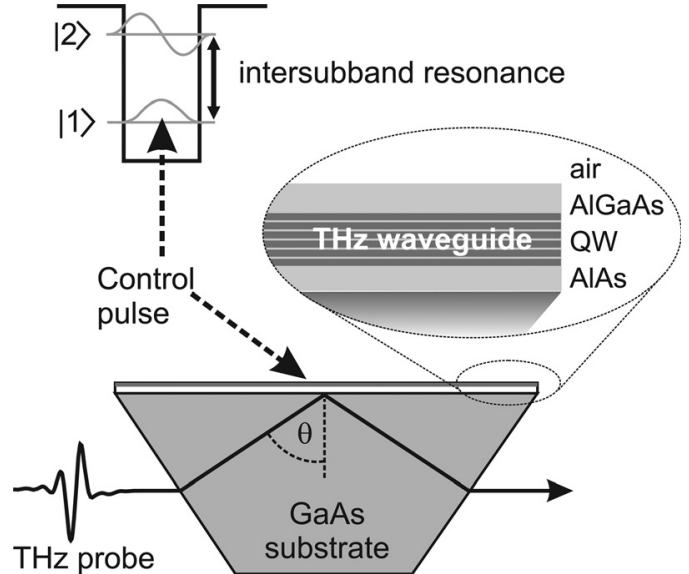

FIG. 1. Schematic of the femtosecond optical switch: 50 undoped GaAs quantum wells of a thickness of $9 \mathrm{~nm}$ each, separated by $\mathrm{Al}_{0.33} \mathrm{Ga}_{0.67} \mathrm{As}$ barriers are embedded into a planar waveguide based on total internal reflection. Intersubband transitions are activated by near-infrared 12-fs control pulses populating level $|1\rangle$. TM-polarized multi-THz transients guided through the prism-shaped substrate are reflected off the waveguide to probe the ultrafast buildup of intersubband cavity polaritons.

provided by static delta doping or electronic injection, ${ }^{5-11}$ both of which are difficult to modulate with high bandwidths. We employ a 12-fs control pulse centered at a photon energy of $1.55 \mathrm{eV}$ to photoinject electrons from the valence band into the lowest conduction subband of the QWs, activating the intersubband oscillator within femtoseconds. Since the inverse frequency of the latter transition amounts to $37 \mathrm{fs}$, switching occurs within less than half a cycle of light.

The subsequent ultrafast dynamics of the nonequilibrium cavity at room temperature is traced by multi-terahertz (THz) spectroscopy: ${ }^{17} \mathrm{~A}$ second part of the laser output generates phase-locked $\mathrm{THz}$ pulses covering the spectral window from 80 to $150 \mathrm{meV}$ by optical rectification in a $50-\mu \mathrm{m}$-thin GaSe emitter. ${ }^{18}$ TM-polarized field transients are coupled through the prism-shaped substrate and internally reflected off the photoexcited area of the waveguide under incidence angles around $\theta=65^{\circ}$ (Fig. 1). The pulse front of the near-infrared pump is tilted by means of a grating to match the geometry of the THz phase surfaces. The oscillating electric field of the reflected $\mathrm{THz}$ transient is resolved in the time domain via phase-matched electro-optic sampling. ${ }^{18}$

Figure 2(a) displays a typical phase-locked few-cycle pulse incident on the microcavity. For a vanishing fluence $\Phi=0$ of the control pulses [Fig. 2(b)], the reflected field exhibits a characteristic twin-pulse profile. This temporal structure may be understood as follows: Part of the incident field is directly reflected off the cavity surface. A second portion is evanescently coupled into the resonator, prepares a coherent photon state, and gets reemitted with a measurable time delay. Hence the initial burst is due to instantly reflected light while the second part results from reemission. The two contributions are also distinguished via their carrier phase: While the instantly reflected portion is precisely in phase with the incident field, the reemitted radiation shows a phase offset by $\pi$ - the hallmark behavior of a resonantly driven harmonic oscillator.

For delay times $T>200 \mathrm{fs}$, the incident THz field preparing the coherent intracavity photon population has com-

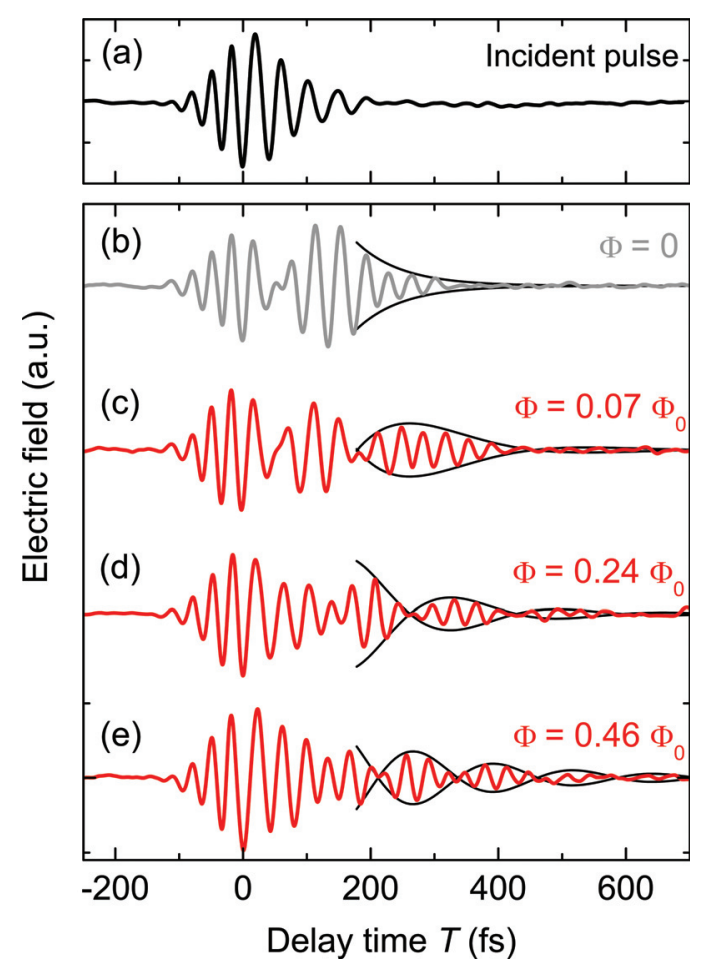

FIG. 2. (Color online) All-optical in situ tuning of cavity polariton beats in the time domain. (a) $\mathrm{THz}$ transient incident on the microcavity structure. (b)-(e) Electric field reflected off the cavity for various control fluences (indicated next to each curve, $\Phi_{0}=0.1 \mathrm{~mJ} \mathrm{~cm}^{-2}$ ). All data are recorded at a fixed delay time $t_{D}=20 \mathrm{ps}$ and at temperature $T_{L}=300 \mathrm{~K}$. (b) The twin pulse structure is due to radiation directly reflected off the cavity (first burst) and bare photons reemitted from the cavity (second pulse). (c)-(e) At finite control fluences, cavity polaritons manifest themselves via a pronounced beating. Thin dotted curves mark the field envelopes of (b) the free cavity decay and (c)-(e) polariton beating, respectively.

pletely vanished. Consequently, the reemitted field follows an exponentially decaying envelope. It is this free cavity decay that is most dramatically affected when light-matter coupling is active: Fig. 2(c)-2(e) display the reflected $\mathrm{THz}$ field at a delay time $t_{D}=20 \mathrm{ps}$ after incidence of the control pulse. At a pump fluence $\Phi=0.07 \times \Phi_{0}$ [Fig. 2(c)] the single-exponential decay is absent. Rather, the reflected field trace shows the onset of beating. As will be discussed below, this feature is the time-domain fingerprint of vacuum Rabi oscillations: Energy is periodically exchanged between the intersubband excitation and the light field, giving rise to characteristic beat nodes. Panels (d-e) illustrate that the beating frequency increases with the pump fluence $\Phi$. The relative strength of light-matter coupling becomes very obvious in this time-domain image: For large control fluences [Figs. 2(d) and 2(e)], only few cycles of the carrier field are observed per Rabi node, confirming the ultrastrong nature of light-matter coupling.

Fourier transformation provides the corresponding amplitude and phase spectra in the mid infrared. The eigenmodes of the cavity are identified via their characteristic minima in the amplitude reflectivity. In Fig. 3(a), we demonstrate that light-matter coupling is continuously tunable via the control fluence $\Phi$. In equilibrium $(\Phi=0)$, a single reflectance minimum at $\hbar \omega_{c}=113 \mathrm{meV}$ (top curve) attests to the sole resonance of the unexcited cavity, the bare photon 
Reflected THz field (a.u.)
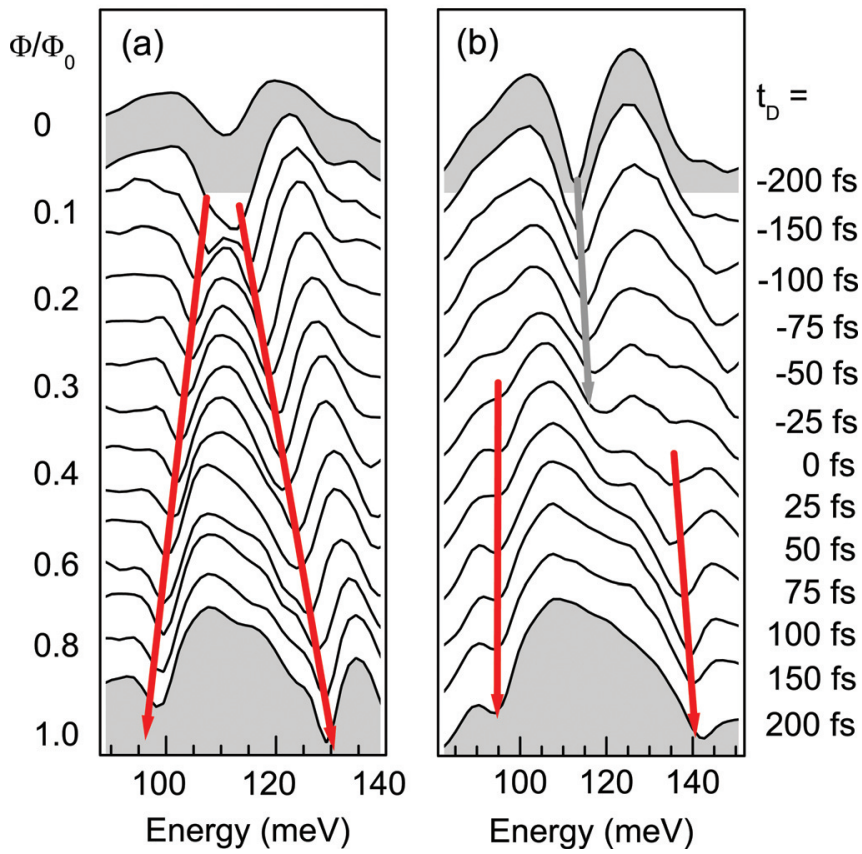

FIG. 3. (Color online) (a) THz reflectance spectra measured at room temperature for various fluences $\Phi$ (vertically offset) of the control pulse $\left(t_{D}=20 \mathrm{ps}\right)$. Minima indicate eigenmodes of the system. For $\Phi=0$, only the bare photon mode is observed at $\hbar \omega_{c}=113 \mathrm{meV}$; both branches of the intersubband cavity polaritons are discernible for $\Phi>0.05 \times \Phi_{0}$, where $\Phi_{0}=0.1 \mathrm{~mJ} / \mathrm{cm}^{2}$. The transition from weak via strong to ultrastrong coupling is conveniently tuned by the control fluence $\Phi$. (b) Two-dimensional optical pump- THz probe data. Amplitude reflectivity spectra at $T_{L}=300$ $\mathrm{K}$ for various delay times $t_{D}$ (control fluence: $\Phi=0.1 \mathrm{~mJ} / \mathrm{cm}^{2}$ ). The arrows serve as guides to the eye for the position of the cavity and polariton resonances, respectively. In contrast to (a) the two polariton modes do not emerge from the bare cavity resonance by adiabatic bifurcation, but via abrupt switching (data from Ref. 16).

mode. For $\Phi>0.05 \times \Phi_{0}, \Omega_{R}$ exceeds the widths (FWHM $\approx 5 \mathrm{meV}$ ) of both intersubband and cavity resonances and two cavity polariton branches are clearly discernible. At this point, the system crosses from the weak to the strong coupling regime. Further increase of the fluence enhances the separation of the minima. The maximal energetic distance of the two polariton modes could be increased to as much as $50 \mathrm{meV}$, in our experiment, corresponding to a fraction of $44 \%$ of the bare photon frequency. The apparent mode separation is not identical with the vacuum Rabi splitting at the anticrossing point. ${ }^{10}$ Only a quantitative simulation of the energy position of the polariton dips allows for extraction of $\Omega_{R}$. For a correct description of our data, the theory has to go beyond the rotating wave approximation and include antiresonant terms in the light-matter Hamiltonian, as discussed in more detail below. By comparison with this theory we determine a maximum splitting $2 \Omega_{R}=0.18 \times \omega_{12}$ for our experiments. This value is comparable to the record achieved in doped structures in the mid infrared ${ }^{8,9}$ and clearly fulfills the criteria for ultrastrong coupling. ${ }^{14}$

The central question is: How rapidly may ultrastrong coupling be activated? In order to address this issue, we repeat the experiments for various delay times $t_{D}$ between near-infrared pump and multi-THz probe pulses. The results are illustrated in Fig. 3(b): For $t_{D}=-50$ fs the spectra are

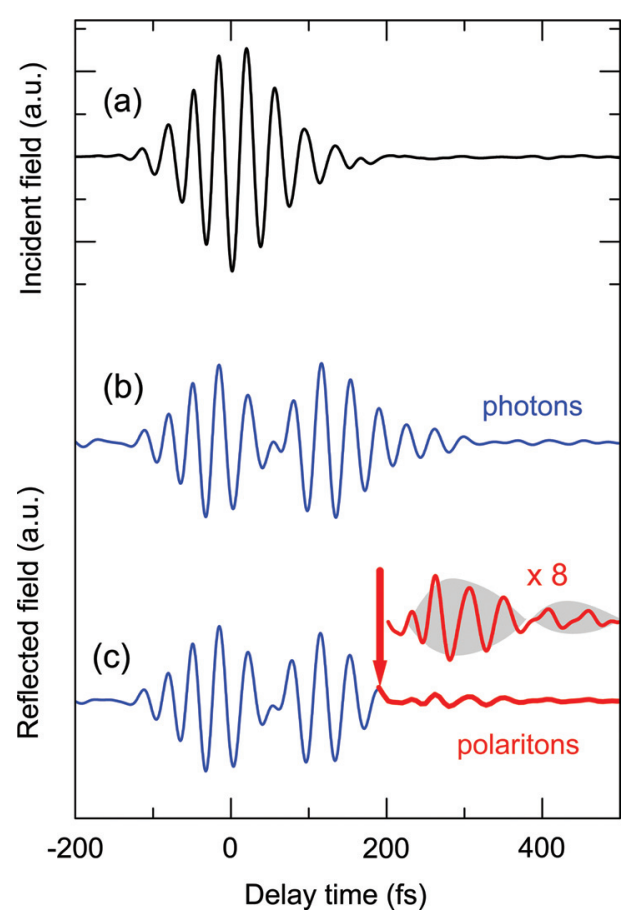

FIG. 4. (Color online) Perturbed cavity decay in the time domain: Bare photons are converted nonadiabatically into cavity polaritons. (a) Incident $\mathrm{THz}$ transient, (b) reflected transient without control pulse. (c) The near-infrared control pulse (vertical arrow) alters the cavity emission on a subcycle scale: The exponential bare cavity decay is replaced by the characteristic polariton beating (insert, magnified by factor of 8 ).

dominated by a single dip located at $113 \mathrm{meV}$ which we may unequivocally assign to the photonic waveguide mode. Photoinjection of electrons into the lower subband induces dramatic changes of the spectra of order unity. The initial bare photon eigenstate is replaced, on a ten femtosecond scale, by two ultrastrongly coupled cavity polariton modes appearing simultaneously at energies of 94 and $143 \mathrm{meV}$, respectively (arrows in Fig. 3). Most remarkably, the new resonances do not develop by gradual bifurcation out of the bare cavity mode. In other words, the system does not sequentially pass through all three regimes of light-matter coupling — from weak to strong and finally ultrastrong interaction, similar to Fig. 3(a). In contrast, switching occurs discontinuously as soon as subband $|1\rangle$ is populated.

Femtosecond activation of the light-matter Hamiltonian is predicted to give rise to yet unexplored effects on preexisting photon states and the quantum vacuum itself. ${ }^{14}$ This class of phenomena is best studied directly in the time domain: Fig. 4(a) and 4(b) illustrate the incident $\mathrm{THz}$ transient and the field reflected off the unexcited cavity, encoding the reflected and reemitted portion of light from the optical waveguide, respectively.

The most intriguing situation is realized if we turn lightmatter coupling on while a coherent state of bare photons is still present inside the cavity: The control pulse [vertical arrow in Fig. 4(c)] abruptly alters $\Omega_{R}$ during the free cavity decay. Remarkably, the emission of bare photons is interrupted on a time scale shorter than half an oscillation cycle of light - a compelling proof of nonadiabaticity. The subsequent field trace exhibits a characteristic two-mode 
beating [inset in Fig. 4(c)], the hallmark of coherent oscillations of both polariton branches. Thus we do not only control the eigenstates of the microcavity, but effectively convert a coherent photon population into ultrastrongly coupled cavity polaritons beating at the splitting frequency. We suggest the term "perturbed cavity decay" (PCD) to describe this novel subcycle phenomenon.

While all the above experiments focus on the dynamics of coherent photon and polariton populations, theory anticipates an exciting new class of unusual quantum correlations to occur in the presented systems, as well. The origin of these novel quantum electrodynamics (QED) phenomena is intimately related with the nature of ultrastrong light-matter coupling, which manifests itself clearly in our data: Fig. 5 displays the experimental dependence of the polariton eigenfrequencies on the pump fluence as extracted from data similar to Fig. 3(a). As mentioned above, it turns out that a quantitative model has to go beyond first order perturbation theory. ${ }^{14}$ We describe the intersubband excitation in a bosonic approximation with a Hopfield-like Hamiltonian. The well-known strong coupling part contains the energy of the bare cavity and the intersubband polarization, as well as the resonant part of the light-matter interaction: Creation (annihilation) of one photon with simultaneous annihiliation (creation) of an intersubband excitation at the same wavevector $k$. Beyond the rotating wave approximation, we also include antiresonant contributions of the form: ${ }^{14}$

$$
\begin{aligned}
H_{\text {anti }}= & \hbar \sum_{k}\left[i \Omega_{R, k}\left(a_{k} b_{-k}+a_{k}^{\dagger} b_{-k}^{\dagger}\right)\right. \\
& \left.+D_{k}\left(a_{k} a_{-k}-a_{k}^{\dagger} a_{-k}^{\dagger}\right)\right] .
\end{aligned}
$$

Here, $a_{k}^{\dagger}\left(a_{k}\right)$ is the creation (annihilation) operator for the fundamental cavity-photon mode with in-plane wavevector $k$ and frequency $\omega_{\mathrm{cav}, k} \cdot b_{k}^{\dagger}$ is the creation operator of the bright intersubband excitation mode, and $\Omega_{R, k}$ is the vacuum Rabi frequency, which may be tuned via the density $N_{e}$ of photoexcited electrons in subband $|1\rangle$ as defined in Eq. (1). The terms proportional to $\Omega_{R, k}$ describe the creation (destruction) of a cavity photon and an intersubband excitation, whereas

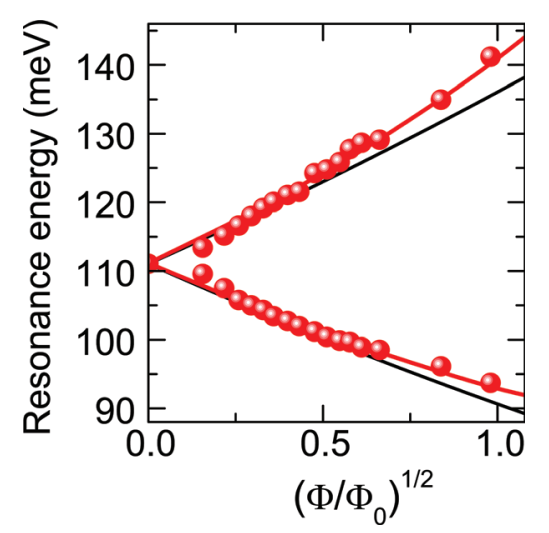

FIG. 5. (Color online) Center frequencies of the lower and upper cavity polariton branches as a function of the control fluence $\left(\Phi_{0}=0.1 \mathrm{~mJ} \mathrm{~cm}^{-2}\right.$, $T_{L}=300 \mathrm{~K}$ ). Dots: experimental data; red curves: theory including antiresonant terms of light-matter coupling; black curves: first-order perturbation theory within the rotating wave approximation (data from Ref. 16). the term proportional to $D_{k}$ contains only photon operators because it originates from the square of the electromagnetic vector potential. Remarkably, the latter contribution describes the simultaneous creation and annihilation of two excitations with opposite in-plane vector $k$. For the case of a deep rectangular QW, $D_{k}$ may be approximated as $D_{k} \approx \Omega_{R}^{2} / \omega_{12},{ }^{14}$ i.e., this contribution becomes appreciable in the regime of ultrastrong coupling. In particular, it leads to unconventional quantum correlations and a two-mode squeezed quantum vacuum.

We find that a theory neglecting $H_{\text {anti }}$ does not reproduce the measured functions appropriately (black line in Fig. 5). In particular, the slowly varying envelope approximation (SVA) predicts a more symmetric splitting of the polariton resonances with respect to the bare photon mode, whereas the experiment suggests a strong asymmetry with the average of both resonances shifting to higher frequencies at higher pump fluences. This effect is mainly caused by quadratic contributions of the vector potential to the light-matter Hamiltonian and may be regarded as the vacuum counterpart of the classical Bloch-Siegert shift. The experiment is well described only after addition of antiresonant terms in the interaction Hamiltonian (red curves threading the experimental data points).

In our optical switch, the ground state is, thus, predicted to be a squeezed vacuum containing a finite number of virtual photons. ${ }^{14}$ As long as the quanta are confined inside the cavity their unconventional nature remains inaccessible. Theory shows, however, that nonadiabatic switching of $\Omega_{R}$ as demonstrated in this paper may release these virtual quanta in correlated pairs. ${ }^{14}$ This phenomenon is only one out of a whole new class of nonadiabatic QED effects reminiscent of the dynamical Casimir effect or Hawking radiation of black holes. ${ }^{25,26}$ A quantitative estimate via the theory of Ref. 14 shows that for the switching times demonstrated here $\left(10^{-14} \mathrm{~s}\right)$ the number of vacuum photons released per pulse should be of the order of $10^{3}$. In fact, latest developments of sensitive phase-matched electro-optic detection, based on extremely stable fiber lasers, is able to detect a number of photons as lows as $10^{2}$ per cycle of light. ${ }^{27} \mathrm{~A}$ direct field resolved observation of photon statistics could, therefore, well become reality in the near future.

The opposite high-intensity limit of light-matter coupling may be systematically investigated, as well: Recently, a novel concept of a source of ultraintense $\mathrm{THz}$ transients has been demonstrated which reaches peak electric $\mathrm{THz}$ fields of up to $100 \mathrm{MV} / \mathrm{cm}$ - values comparable with intraatomic potential gradients. ${ }^{28}$ Exposing the current cavity polariton system to such high-energy coherent $\mathrm{THz}$ fields opens up the unique possibility to study light-matter coupling at the transition from pure quantum vacuum to coherent classical conditions. Corresponding experiments are under way.

In conclusion, we have demonstrated the first nonadiabatic switching scheme of cavity-polaritons, reaching the regime of ultrastrong light-matter coupling on a subcycle scale. The experiments provide a benchmark for latest theories in the ultrastrong coupling regime, point out a viable route toward novel QED phenomena such as the observation 
of Casimir-type vacuum radiation, and demonstrate a roomtemperature optical switching device at the ultimate speed.

This work has been supported by the Deutsche Forschungsgemeinschaft via the Emmy Noether Program and SFB767. A.A.A acknowledges support by the Alexander von Humboldt Foundation.

${ }^{1}$ J. M. Raimond, M. Brune, and S. Haroche, Rev. Mod. Phys. 73, 565 (2001). ${ }^{2}$ F. Brennecke, T. Donner, S. Ritter, T. Bourdel, M. Kohl, T. Esslinger, Nature 450, 268 (2007).

${ }^{3}$ C. Weisbuch, M. Nishioka, A. Ishikawa, and Y. Arakawa, Phys. Rev. Lett. 69, 3314 (1992).

${ }^{4}$ J. P. Reithmaier, G. Sęk, A. Löffler, C. Hofmann, S. Kuhn, S. Reitzenstein, L. V. Keldysh, V. D. Kulakovskii, T. L. Reinecke, and A. Forchel, Nature 432, 197 (2004).

${ }^{5}$ D. Dini, R. Köhler, A. Tredicucci, G. Biasiol, and L. Sorba, Phys. Rev. Lett. 90, 116401 (2003).

${ }^{6}$ L. Sapienza, A. Vasanelli, R. Colombelli, C. Ciuti, Y. Chassagneux, C. Manquest, U. Gennser, and C. Sirtori, Phys. Rev. Lett. 100, 136806 (2008).

${ }^{7}$ S. I. Tsintzos, N. T. Pelekanos, G. Konstantinidis, Z. Hatzopoulos, and P. G. Savvidis, Nature 453, 372 (2008).

${ }^{8}$ A. A. Anappara, S. De Liberato, A. Tredicucci, C. Ciuti, G. Biasiol, L. Sorba, and F. Beltram, Phys. Rev. B Rapid 79, 201303 (2009).

${ }^{9}$ Y. Todorov, A. M. Andrews, I. Sagnes, R. Colombelli, P. Klang, G. Strasser, and C. Sirtori, Phys. Rev. Lett. 102, 186402 (2009).

${ }^{10}$ A. A. Anappara, A. Tredicucci, G. Biasiol, and L. Sorba, Appl. Phys. Lett. 87, 051105 (2005); A. A. Anappara, A. Tredicucci, F. Beltram, G. Biasiol, and L. Sorba, Appl. Phys. Lett. 89, 171109 (2006).

${ }^{11}$ E. Dupont, J. A. Gupta, and H. C. Liu, Phys. Rev. B 75, 205325 (2007).

${ }^{12}$ B. Peropadre, P. Forn-Díaz, E. Solano, and J. J. García-Ripoll, Phys. Rev. Lett. 105, 023601 (2010).
${ }^{13}$ T. Niemczyk, F. Deppe, H. Huebl, E. P. Menzel, F. Hocke, M. J. Schwarz, J. J. Garcia-Ripoll, D. Zueco, T. Hümmer, E. Solano, A. Marx, and R. Gross, Nature Phys. 6, 772 (2010).

${ }^{14}$ C. Ciuti, G. Bastard, and I. Carusotto, Phys. Rev. B 72, 115303 (2005); S. De Liberato, C. Ciuti, I. Carusutto, Phys. Rev. Lett. 98, 103602 (2007).

${ }^{15}$ E. Yablonovitch, Phys. Rev. Lett. 62, 1742 (1989).

${ }^{16}$ G. Günter, A. A. Anappara, J. Hees, A. Sell, G. Biasiol, L. Sorba, S. De Liberato, C. Ciuti, A. Tredicucci, A. Leitenstorfer, and R. Huber, Nature 458, 178 (2009).

${ }^{17}$ R. Huber, F. Tauser, A. Brodschelm, M. Bichler, G. Abstreiter, and A. Leitenstorfer, Nature 414, 286 (2001); R. Huber, C. Kübler, S. Tübel, A. Leitenstorfer, Q. T. Vu, H. Haug, F. Köhler, and M.-C. Amann, Phys. Rev. Lett. 94, 027401 (2005); C. Kübler, H. Ehrke, R. Huber, R. Lopez, A. Halabica, R. F. Haglund, Jr., and A. Leitenstorfer, Phys. Rev. Lett. 99, 116401 (2007).

${ }^{18}$ C. Kübler, R. Huber, S. Tübel, and A. Leitenstorfer, Appl. Phys. Lett. 85, 3360 (2004); C. Kübler, R. Huber, and A. Leitenstorfer, Semicond. Sci. Technol. 20, 128 (2005) and references therein.

${ }^{19} \mathrm{M}$. Tonouchi, Nature Photon. 1, 97 (2007) and references therein.

${ }^{20}$ B. Ferguson and X.-C. Zhang, Nature Mater. 1, 26 (2002) and references therein.

${ }^{21}$ J. Kröll, J. Darmo, S. S. Dhillon, X. Marcadet, M. Calligaro, C. Sirtori, and K. Unterrainer, Nature 449, 698 (2007).

${ }^{22}$ P. Gaal, W. Kuehn, K. Reimann, M. Woerner, T. Elsaesser, and R. Hey, Nature 450, 1210 (2007).

${ }^{23}$ N. Jukam, S. S. Dhillon, D. Oustinov, J. Madeo, C. Manquest, S. Barbieri, C. Sirtori, S. P. Khanna, E. H. Linfield, A. G. Davies, and J. Tignon, Nature Photon. 3, 715 (2009).

${ }^{24} \mathrm{M}$. Helm in Intersubband Transitions in Quantum Wells: Physics and Device Applications I, Semiconductors and Semimetals, edited by H. C. Liu and F. Capasso (Academic, San Diego, 2000), Vol. 62.

${ }^{25}$ W. G. Unruh, Phys. Rev. D 10, 3194 (1974).

${ }^{26}$ S. W. Hawking, Nature 248, 30 (1974).

${ }^{27}$ A. Sell, R. Scheu, A. Leitenstorfer, and R. Huber, Appl. Phys. Lett. 93, 251107 (2008).

${ }^{28}$ A. Sell, A. Leitenstorfer, and R. Huber, Opt. Lett. 33, 2767 (2008). 